\title{
Le rôle de la L1 dans le français du Mali : une étude perceptive
}

\author{
Chantal Lyche \\ Université d'Oslo, Norvège \\ chantal.lyche@ilos.uio.no \\ Ingse Skattum \\ Université d'Oslo, Norvège \\ ingse.skattum@ikos.uio.no
}

\section{Introduction}

Dans ce travail, nous nous proposons d'examiner l'impact de la langue première (L1) sur l'accent en français langue seconde (FLS), dans le sens de langue officielle et / ou langue d'enseignement, mais non L1 des populations ni langue étrangère ordinaire (Cuq, 1991). Dans une étude entreprise à Bamako, au Mali, nous nous concentrerons plus particulièrement sur l'aptitude des locuteurs à détecter la L1 de leurs compatriotes, sur les caractéristiques qu'ils considèrent comme les plus discriminantes et sur les facteurs propices au maintien ou au nivellement de l'accent.

Les tests de perception constituent une branche relativement récente de la sociolinguistique, développée à partir des années 1980 (Boughton, 2006 : 277). La dialectologie perceptive s'attache à déterminer les caractéristiques, réelles ou imaginaires, souvent stéréotypées, des accents, en alliant l'étude des représentations linguistiques à la variation diatopique (géographique), souvent aussi diastratique (sociale), et parfois diaphasique (stylistique) - mais plus rarement interférentielle (provenant d'un substrat ou d'un adstrat). Les linguistes savent que la notion de variété est une construction et que «le découpage ainsi supposé ne résiste pas à l'observation des productions effectives, qui peuvent être souples, labiles, et plus souvent hétérogènes qu'homogènes » (Gadet, 2007: 22). Or, les tests de perception permettent de comparer la catégorisation opérée par les chercheurs aux représentations des usagers, d'une part et, d'autre part, aux données objectives des mesures acoustiques. A partir de telles comparaisons il est possible d'identifier les facteurs phonologiques et sociolinguistiques qui fondent ces «découpages » de part et d'autre. Parmi les variables les plus souvent testées, on note l'enracinement géographique, l'âge et le sexe et, en ce qui concerne la L2, aussi l'âge d'appropriation de la L2, la motivation et l'aptitude de l'apprenant, l'exposition à la L2 et le degré d'utilisation de la L1 (cf. par ex. Guion, Flege et Loftin, 2000 ; Piske, MacKay et Flege, 2001).

La perception des accents du français (L1 ou FLE - français langue étrangère) a été examinée inter alia par Bauvois (1996), Boughton (2006), Woehrling et Boula de Mareüil (2006) et Vieru-Dimulescu et Boula de Mareüil (2006). Peu d'études ont porté sur l'identification des accents en français langue seconde (FLS) dans le sens cité (voir cependant Féral, 1979 ; Prignitz, 1994 ; Moreau, Thiam et Bauvois, 1998). Les variables testées sur le français varient selon les études, les paramètres de base étant l'enracinement géographique, l'âge et le sexe. L'origine géographique est testée et trouvée pertinente dans plusieurs études, dont deux qui lui substituent la L1. Vieru-Dimulescu et Boula de Mareüil montrent que la distance typologique entre la L1 des locuteurs et le français jouent en faveur de la reconnaissance de l'accent étranger : à degré d'accent égal, l'arabe s'identifie par exemple plus aisément que les langues romanes. Dans le domaine africain, Moreau et al. concluent que les Sénégalais reconnaissent difficilement la L1 de leurs compatriotes à travers l'accent en français. L'âge s'avère pertinent dans certains tests, mais n'est pas intégré dans tous. Le sexe est sans impact dans la plupart des 
études. Bauvois sélectionne ses locuteurs aussi en fonction de leur niveau de scolarité, sans toutefois le mettre à l'épreuve. Les deux paramètres urbain/rural et classe sociale sont analysés par deux des tests seulement, Boughton et Moreau et al. Or, comme le remarquent Woehrling et Boula de Mareüil (2006: 86), «il est possible qu'aujourd'hui les frontières socio-culturelles priment sur les frontières géographiques, contrairement à ce qui est traditionnellement dit du français ».

Dans cette étude sur le FLS au Mali, nous envisagerons l'interaction des facteurs phonétiques et sociolinguistiques qui interviennent dans l'identification de la L1 des locuteurs. Il s'agira dans un premier temps de confronter au signal acoustique les traits perçus comme discriminants par les auditeurs. Dans un deuxième temps, nous mettrons en rapport les taux d'identification des L1 avec les caractéristiques sociodémographiques des locuteurs. Ce travail s'articulera comme suit: nous survolerons en (2) la situation sociolinguistique du Mali avant de présenter en (3) la méthodologie du test. Nous étudierons en (4) les traits phonologiques que les auditeurs associent à la notion d'accent avant de prendre en considération en (5) les facteurs sociolinguistiques qui participent au maintien ou au nivellement des traces de la L1.

\section{Situation sociolinguistique au Mali}

A l'indépendance en 1960, la quasi-totalité des anciennes colonies françaises et belges gardèrent le français comme langue officielle et langue d'instruction, bien qu'il soit parlé par une minorité de la population seulement. Au Mali, il s'agit aujourd'hui de 5 à $10 \%$ de celle-ci, l'un des taux les plus faibles en Afrique subsaharienne (Rossillon, 1995). Cette situation s'explique d'abord par l'existence d'une langue endogène dominante au niveau national, le bambara (parlé par environ $80 \%$ de la population comme L1 ou L2), qui assume le rôle de lingua franca et qui de ce fait confine le français au domaine formel. Y contribue aussi le taux très faible d'alphabétisation (22,9\% en 2009, L'état de l'Afrique 2009), l'appropriation du français passant essentiellement par l'école. Le contact restreint avec les Français ainsi que le statut très fort de la tradition concourent également à la préservation des langues du terroir et à la défense du plurilinguisme, affirmée par la Constitution et largement approuvée par la population (cf. Skattum, 2008).

Le Mali compte environ 14 millions d'habitants L'état de l'Afrique 2009), parlant une vingtaine de langues endogènes. Parmi ces langues, 13 sont déclarées « langues nationales » (LN) (Canut et Dumestre 1993 : 220), statut qui implique leur codification, avec un alphabet et des règles d'orthographe. Elles appartiennent à trois grandes familles de langues africaines : la famille Niger-Congo, la famille nilosaharienne et la famille afro-asiatique (chacune représentée dans les données de notre test). La famille Niger-Congo en est la plus importante, au Mali comme à l'échelle continentale. Elle est représentée au Mali par trois groupes linguistiques : mandé (bambara, malinké, khassonké, soninké, bozo), atlantique (fulfulde) et gur (sénoufo, minyanka, bomu). La famille nilo-saharienne est représentée par le songhay ${ }^{1}$, et la famille afro-asiatique par le tamasheq et le hassaniya. La dernière des LN, le dogon, n'est pas encore classifiée. Malgré l'égalité de principe des LN, leur usage varie en fonction de la véhicularité, du nombre de locuteurs, de la variation dialectale et de la parenté des langues ainsi que du poids social des communautés. Ainsi, outre le bambara, originaire du sud, le Mali compte 3 langues véhiculaires régionales : le songhay au nord, le fulfulde (peul) au centre et le soninké à l'ouest. Pour des raisons politiques, le tamasheq, autre langue du nord (parlée par les Touareg), fut l'une des 4 premières LN à bénéficier d'une codification et à être utilisée comme moyen d'instruction.

\section{Méthodologie}

Le test au Mali s'inscrit dans le cadre du projet Contemporary French in Africa : usage, varieties and structures (CFA) associé au projet Phonologie du français contemporain : usage, variétés et structure $(\mathrm{PFC})^{2}$. CFA adopte le protocole PFC (Durand et Lyche, 2003 ; Boutin, Lyche et Prignitz, 2007), en le complétant pour permettre des analyses syntaxiques et sociolinguistiques ciblées, et en l'adaptant au contexte plurilingue (Lyche et Skattum, 2009). Au Mali, 47 locuteurs ont été enregistrés selon le protocole PFC, c'est-à-dire que tous les locuteurs ont été soumis à deux tâches de lecture (liste de mots, 
texte) et qu'ils se sont prêtés à deux types de conversation (entretien semi-directif, conversation libre entre 2 personnes se connaissant). La sélection des témoins s'est faite selon quatre paramètres qui sont, par ordre d'importance décroissante : 1) le niveau d'instruction; 2) l'âge; 3) la L1;4) le sexe.

Pour mener à bien le test de perception, qui s'est déroulé en janvier 2009 à partir des enregistrements recueillis en décembre 2006, 14 locuteurs parmi les 47 enregistrés ont été sélectionnés. L'âge et le sexe s'étant révélés sans signification pour ce test ${ }^{3}$, ils ne seront pas pris en compte ici - pas plus que le plurilinguisme, pourtant frappant (cf. le tableau 1).

Le niveau d'études, facteur essentiel pour les compétences en français dans le contexte africain (Manessy, 1994 : 206, Queffélec, $2008: 67$ ), est défini en fonction des diplômes obtenus aux trois niveaux scolaires en vigueur au Mali ${ }^{4}$ : 1) CEP (Certificat d'études primaires) $=6$ ans de parcours scolaire ;2) DEF (Diplôme d'études fondamental) $=9$ ans ; 3) $\mathrm{Bac} / \mathrm{Bac}^{+}=12$ ans, plus éventuellement des études supérieures.

Les $5 \mathrm{~L} 1 \mathrm{du}$ test ont été choisies en fonction de leur représentativité typologique et de leur importance sociolinguistique : bambara (groupe mandé), fulfulde (groupe atlantique), sénoufo (groupe gur), toutes de la famille Niger-Congo, songhay (famille nilo-saharienne) et tamasheq (famille afro-asiatique).

Le tableau 1 présente les 14 locuteurs dans l'ordre d'écoute et montre leur profil social selon les 4 paramètres précités. Les langues parlées sont données selon l'ordre de maîtrise déclarée et les niveaux d'études, standardisés, sont spécifiés entre parenthèses :

Tableau 1. Les 14 locuteurs selon les 4 paramètres sociaux, dans l'ordre d'écoute

\begin{tabular}{|c|c|c|}
\hline $\begin{array}{l}\text { Loc. : } \\
\text { ordre } \\
\text { (âge) sexe }\end{array}$ & $\begin{array}{l}\text { L1 (en gras), L2, L3... } \\
\text { (+ langue(s) non testée(s)) }\end{array}$ & Niveau d'études \\
\hline $1(57) \mathrm{F}$ & SE, BA. FR & DEF (+2, CAP, jardin d'enfants) \\
\hline $2(51) \mathrm{F}$ & SE, BA, FR & Bac (DEF+4, comptabilité) \\
\hline $3(36) \mathrm{F}$ & TA, SO, FR, BA & DEF ( 9 ans sans dipl.) \\
\hline $4(68) \mathrm{H}$ & BA, FU, FR, SO + 2 Langues & $\mathrm{Bac}$ \\
\hline $5(22) \mathrm{H}$ & SO, FR, BA & Bac (+2, Lettres) \\
\hline $6(62) \mathrm{F}$ & BA, $F R+2 L$ & Bac $(+2+3$, secrét. médical + secr. spéc. $)$ \\
\hline $7(53) \mathrm{F}$ & TA, FR, BA & CEP \\
\hline $8(50) \mathrm{F}$ & FU/FR à égalité, BA, $\mathrm{SO}+1 \mathrm{~L}$ & DEA ( +2 ans de recherche, lettres) \\
\hline $9(24) \mathrm{H}$ & SO, TA, FR. BA + 2 L & Bac (+1, socio-anthropologie $)$ \\
\hline $10(46) \mathrm{H}$ & BA, FR & CEP (+2 sans diplôme) \\
\hline $11(49) \mathrm{H}$ & SO, BA, FR & DEF $(+2$, lycée technique sans dipl.) \\
\hline $12(30) \mathrm{F}$ & TA, FR. BA, SO & DEF (CEP +4 , coupe et couture) \\
\hline $13(62) \mathrm{H}$ & BA, FR & CEP \\
\hline $14(54) \mathrm{H}$ & FU, FR, BA, + 1 L & Thèse (pédagogie) \\
\hline
\end{tabular}

Légende : $\mathrm{BA}=$ bambara, $\mathrm{FR}=$ français, $\mathrm{FU}=$ fulfulde, $\mathrm{SE}=$ sénoufo, $\mathrm{SO}=$ songhay, $\mathrm{TA}=$ tamasheq

Un extrait d'une minute de parole tiré de l'entretien semi-directif a été soumis à six auditeurs maliens âgés de 18 à 60 ans qui, parmi les langues testées, parlent le bambara et le fulfulde. Trois de ces auditeurs sont qualifiés d' « experts » du fait de leur formation linguistique. 
La passation du test a consisté en l'écoute des extraits d'une minute de parole spontanée, qui étaient sans indices sur l'origine ethnique ou linguistique du locuteur. L'ordre des locuteurs était aléatoire, alors que l'ordre d'écoute était fixe (cf. le tableau 1). Les auditeurs ont passé le test en présence de l'enquêteur dans une pièce calme mais pas en chambre sourde, avec la possibilité de réécouter les extraits. A la fin de chaque extrait, ils répondaient aux questions ci-dessous et leurs réponses ont été enregistrées pour mener à bien l'analyse. Les auditeurs n'avaient pas la liste des langues testées; leur choix pouvait donc a priori porter sur une vingtaine de langues, mais seules 8 ont en fait été proposées : le dogon, le khassonké et le bomu, en plus des 5 langues du test.

1. Pensez-vous pouvoir identifier la langue maternelle d'un locuteur à travers son français ?

2. Quelle est, selon vous, la langue maternelle du locuteur no. $1,2, \ldots$ ?

3. Pouvez-vous justifier votre réponse?

Après le test proprement dit, une conversation libre portait sur les particularités des accents au Mali et dans les pays voisins. La durée du test (y compris la conversation libre) variait de 16 à $53 \mathrm{mn}$., les « experts » offrant des commentaires plus fournis que les «naïfs ».

\section{Aspects phonologiques}

\subsection{Caractéristiques phonologiques des L1 et remarques des auditeurs}

Les cinq langues nationales choisies pour le test se répartissent géographiquement de la façon suivante : deux langues du nord (songhay et tamasheq), deux langues du sud (bambara et sénoufo) et une langue du centre (fulfulde). Nous ne saurions envisager en détail chacune de ces langues, mais retiendrons dans un premier temps les traits qui les distinguent du français, le plus saillant concernant la prosodie, avec deux langues à tons (les langues du sud, bambara et sénoufo) et trois langues à accent contrastif (fulfulde, songhay et tamasheq) $)^{5}$. Du point de vue segmental, les cinq langues ont en commun de ne pas connaître les voyelles antérieures arrondies comme cela est la norme sur le continent africain, mais elles varient en ce qui concerne les oppositions de hauteur ou d'aperture. Le songhay et le tamasheq (Nicolaï, 1981 ; Heath, 2005) ont un système à cinq voyelles sans allophones ouverts alors que les autres langues connaissent deux degrés d'aperture (contrastifs ou non) pour les voyelles moyennes (Dumestre, 2003, Carlson, 1994 ; Cissé, 2009). Les systèmes consonantiques varient considérablement d'une langue à l'autre et s'opposent au français en ce que ni le bambara ni le fulfulde ne possèdent de fricative voisée pas plus que l'alvéo-palatale /S/. La consonne R lorsqu'elle appartient au système phonologique (ce qui n'est pas le cas pour le sénoufo (Carlson, 1994)) est le plus souvent décrite comme une vibrante dentoalvéolaire (/r/), mais certaines langues alternent également entre occlusives vélaires et la fricative uvulaire (Dumestre, 2003, pour le bambara, Carlson, 1994, pour le sénoufo) selon les environnements. L'alternance peut également avoir lieu entre une battue et une occlusive dentale (Carlson, 1994). Signalons enfin que les structures syllabiques opposent le tamasheq et le songhay, qui autorisent largement les syllabes CVC ou même des codas complexes, aux autres langues qui favorisent les syllabes ouvertes et se distinguent plutôt au niveau de la structure des attaques (Cissé, 2009).

Sur la base de ces traits succincts, nous pouvons déjà dégager quelques pistes pour une différenciation des locuteurs sur la base de leur L1. Les locuteurs des deux langues du nord (songhay et tamasheq) ont en partage le même système vocalique et devraient éprouver des difficultés dans la maîtrise des oppositions d'aperture des voyelles moyennes, mais grâce à la présence dans leurs langues de groupes consonantiques, ils devraient pouvoir prononcer les codas et attaques complexes. Enfin, leur R devrait être moins sujet à variation et être réalisé majoritairement comme une vibrante dento-alvéolaire. Les bambarophones et fulaphones devraient apparaître à leurs compatriotes comme éprouvant des difficultés à prononcer correctement les fricatives du français, aussi bien la série voisée que l'alvéo-palatale sourde. De surcroît, bambarophones et sénoufophones devraient se distinguer par une intonation particulière liée à l'habitude de la pratique des tons. Les remarques générales des auditeurs concordent largement avec ces 
prédictions et parmi les traits mentionnés le plus souvent, on trouve le non arrondissement des voyelles, la réalisation du $\mathrm{R}$ et l'accent / intonation (sans qu'une distinction soit établie entre les deux). Dans leurs caractéristiques des langues du nord, les auditeurs incluent cependant une difficulté à prononcer des voyelles antérieures arrondies ( ils prononcent [parske] pour parce que ») alors que nous avons vu qu'aucune des langues testées ne comporte ces voyelles dans son inventaire. Nous nous tournons à présent vers les performances des locuteurs afin de séparer la réalité de l'imaginaire.

\subsection{Les performances des locuteurs}

\subsubsection{Les voyelles antérieures}

Nous avons choisi de nous concentrer ici sur le système vocalique des locuteurs, sur la prononciation du $\mathrm{R}$ et sur les phénomènes intonatifs. Afin d'obtenir un aperçu plus global des systèmes individuels, nous avons étudié la liste de mots de chaque locuteur, des passages des deux conversations en plus de l'extrait d'une minute qui, rappelons-le, était la seule source fournie aux auditeurs. Sur ces bases nous avons pu établir que les voyelles antérieures arrondies $/ \mathrm{y}, \varnothing /$ posent des difficultés à la grande majorité des locuteurs et que les locuteurs qui les maîtrisent le mieux, 4, 8, 14, ont le bambara (loc. 4) et le fulfulde (loc. 8, 14) comme L1. Il n'en reste pas moins vrai que dans les extraits, les locuteurs 5 et 9 (songhay) prononcent presque systématiquement /i/ pour / $/$ /, alors que les trois locuteurs de tamasheq ont des performances plus variées à ce niveau. Nous verrons en 5 que ces différences peuvent provenir des profils sociolinguistiques.

La distinction voyelle antérieure arrondie / non arrondie dépend étroitement du contexte prosodique : elle est très peu maitrisée en syllabe inaccentuable, mais beaucoup mieux en syllabe accentuable, donc en finale de mot ou de groupe, un contexte où la voyelle est toujours perceptivement plus saillante. On relève ainsi dans la liste de mots les réalisations [peti] petit et [reljyr] reliure, aussi bien chez la loc. 1 (sénoufo) que chez les locuteurs des langues du nord. Dans les conversations ou dans les extraits, tous les locuteurs sans aucune exception prononcent par exemple [e] dans toutes les formes du verbe venir où la voyelle de la syllabe initiale est un schwa ([venir] pour venir). Ces formes exhibent une telle régularité que l'on pourrait envisager qu'elles sont en fait lexicalisées. En ce qui concerne les voyelles antérieures arrondies (fermée et mi-fermée), nous concluons que si tous les locuteurs éprouvent une certaine difficulté à arrondir les voyelles, les locuteurs du nord les mieux reconnus ont un taux de réalisation de voyelles antérieures arrondies inférieur à celui des locuteurs des trois autres langues et que les affirmations des auditeurs se trouvent ainsi confirmées. Les extraits soumis à évaluation sont entièrement conformes à ces remarques, et chez le loc. 9 par exemple, étude(s) est systématiquement réalisé [etid].

La voyelle antérieure arrondie mi-ouverte /œ/ pose aux locuteurs de tamasheq et songhay un double défi, celui de l'arrondissement qui se superpose à celui de l'aperture alors que seul l'arrondissement est problématique pour les autres locuteurs. La prononciation de cette voyelle ne permet pas cependant de séparer les locuteurs sur la base de leur L1. Chez la quasi-totalité des témoins, la voyelle de type Ø suivie d'un R de coda est ouverte [œ], mais souvent fermée dans les autres contextes (par ex. peuple [pøpl]), et les paires jeune-jeûnes sont le plus souvent inversées ([3øn-zœn] respectivement). Les réalisations de la paire jeune-jeûne reflètent probablement l'acquis de l'école où l'accent circonflexe est présenté comme une marque graphique d'ouverture de la voyelle, phénomène qui se retrouve dans d'autres variétés africaines de français (Boutin et Turcsan, 2009). On observe une stratégie identique pour la voyelle de type $\mathrm{O}$, ce qui se traduit dans la liste de mots par la prononciation [njol] pour gnôle.

Les oppositions d'aperture /e- $\varepsilon /$ sont relativement bien acquises par tous les locuteurs, que leur L1 comporte 5 ou 7 voyelles orales. Lorsque les auditeurs mentionnent les difficultés des locuteurs de songhay ou tamasheq à opposer /e- $\varepsilon$ /, nous avons mesuré dans les extraits toutes les valeurs du F1 (premier formant, qui rend compte du degré d'aperture de la voyelle) des voyelles E en syllabe finale de mot. Les locuteurs du nord opposent systématiquement les deux timbres, mais des études acoustiques 
seraient nécessaires pour déterminer si globalement, les systèmes vocaliques de ces locuteurs sont plus fermés que ceux des locuteurs du sud. Nous reviendrons en 5 sur le cas des locuteurs du fulfulde.

\subsubsection{Le R}

Parmi les commentaires récurrents proposés par les auditeurs, nous trouvons également la prononciation $\mathrm{du}$ R. Cependant, tous les ouvrages consultés décrivent le phonème $\mathrm{R}$ dans toutes les langues confondues comme une vibrante dento-alvéolaire. Un examen sous praat (http://www.fon.hum.uva.nl/praat/) des $\mathrm{R}$ prononcés dans les extraits met en évidence que les locuteurs de songhay et de tamasheq réalisent les $\mathrm{R}$ très majoritairement comme une vibrante dento-alvéolaire alors que les locuteurs de bambara, sénoufo et fulfulde alternent régulièrement entre une vibrante dento-alvéolaire, une battue et une approximante uvulaire. Le R des locuteurs de songhay et de tamasheq semble en effet plus distinct, plus prononcé, ce qui pourrait être relié au nombre de vibrations. Nous pensons cependant que la source de cette saillance provient d'un autre phénomène, lié quant à lui à la structure syllabique. Nous savons en effet que dans le français d'Afrique (par ex. Boutin et Turcsan, 2009), tout comme dans les créoles à base française, et dans les variétés de français de locuteurs bilingues français-créole (Bordal, 2006), le R est très souvent sujet à élision. Ce phénomène s'explique en partie par une tendance à favoriser la structure $\mathrm{CV}$, et à interdire les groupes consonantiques complexes, deux caractéristiques communes à de nombreuses langues avec lesquelles le français se trouve en contact. Dans toutes ces variétés de français, le R est absent en position de coda interne ou finale de mot ([pale] parlé, [pu] pour) et parfois même dans les attaques complexes à l'initiale d'un mot ([twa] trois). Dans les groupes obstruante $+\mathrm{R}$ en finale de mot, tout comme dans les variétés européennes, le R est également très faible ([met] mettre). La disparition du $\mathrm{R}$ de coda entraîne régulièrement un allongement compensatoire ou laisse une très légère trace vocalique sous la forme d'un schwa.

Le français du Mali se conforme tout à fait à ces observations et tous les locuteurs sans exception ne prononcent pas le $\mathrm{R}$ dans parler ([pale]), mais au-delà de ce seul mot, les degrés de chute varient considérablement. Les locuteurs dont la L1 est soit le songhay, soit le tamasheq se distinguent aisément des autres locuteurs en ce qu'ils maintiennent massivement les R. Chez par exemple la loc. 7 (tamasheq), tous les $\mathrm{R}$ sont réalisés que ce soit en coda (parti [parti]) ou même dans un groupe final de mot (théâtre prononcé [teatr] lors de ses deux occurrences) alors que dans ce dernier cas, la simplification du groupe est la norme dans la plupart des variétés du français. Chez les locuteurs de songhay et de tamasheq, les $\mathrm{R}$ sont uniquement susceptibles de tomber dans des mots de grande fréquence lexicale, comme parler, peutêtre, parce que et ce phénomène les distingue nettement des autres locuteurs chez qui la chute du $\mathrm{R}$ est conforme à ce qui est décrit dans la littérature : par exemple [ga:sõ] garçon, [3a:dinєr] jardinière chez la loc. 1 (sénoufo).

Cette différence de traitement du $\mathrm{R}$ trouve une explication naturelle dès que l'on envisage la L1 des locuteurs. Le songhay et le tamasheq sont deux langues où les structures $(\mathrm{C}) \mathrm{CVC}(\mathrm{C})$ sont bien intégrées et, de ce fait, les locuteurs maintiennent en français aussi bien les codas que les groupes complexes. Les groupes consonantiques, tout comme les R en coda, sont ainsi maintenus par les locuteurs de songhay et tamasheq alors que les locuteurs des autres langues imposent au français la structure syllabique CV de leur L1. Le R devient alors un trait discriminant pour les locuteurs de songhay et de tamasheq par sa présence massive tout comme par son articulation fort peu variée et rarement affaiblie.

\subsubsection{La prosodie}

La prosodie constitue également un trait majeur intervenant dans la perception d'accent et la détermination de la L1 si l'on se fie aux commentaires des locuteurs. Qu'il en soit ainsi découle directement de la disparité des systèmes brièvement évoquée en 4.1. Les locuteurs doivent acquérir un système où l'accent remplit un rôle démarcatif (le français) alors qu'ils possèdent soit un système où l'accent a un rôle contrastif (tamasheq, songhay, fulfulde), soit un système tonal (bambara, sénoufo). On est donc en mesure de s'attendre à ce que, comme cela a été montré dans des expériences de Boula de 
Mareüil et Vieru-Dimulescu (2006) pour des L2, la prosodie de certains apprenants reste calquée sur celle de leur L1. L'analyse de la $F_{0}$ des extraits de nos locuteurs est révélatrice à cet égard : les deux locuteurs les mieux reconnus (la loc. 7 de langue tamasheq et le loc. 13 de langue bambara) exhibent des profils mélodiques très divergents et maintiennent en français la prosodie de leur L1.

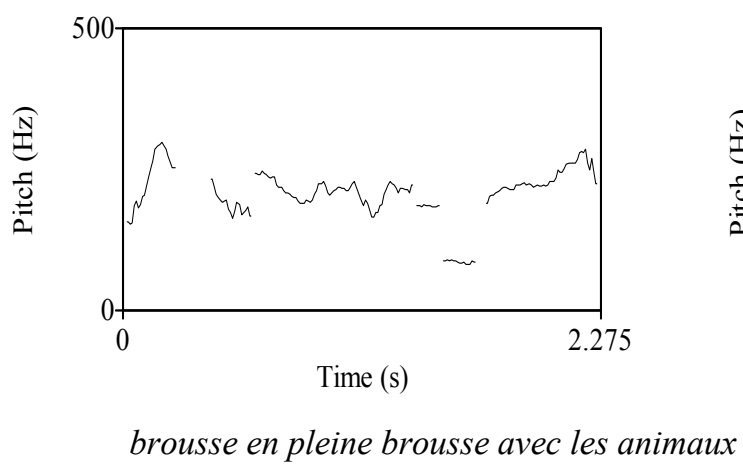

Figure 1. Locutrice 7 (tamasheq)

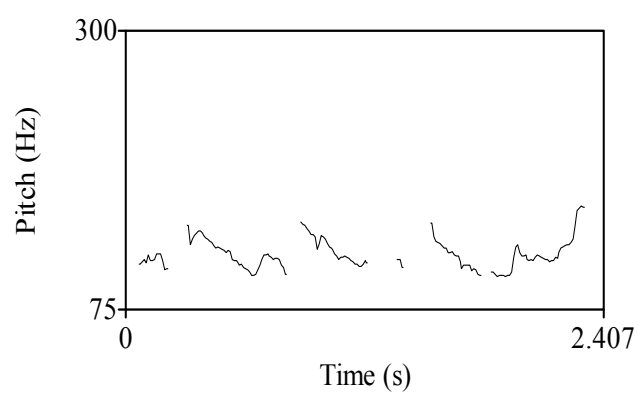

il m'a pris la voiture et puis il l'a vendue

Figure 2. Locuteur 13 (bambara)

La figure 1 montre pour la locutrice tamasheq (langue à accent contrastif) une proéminence avec un sommet de la $\mathrm{F}_{0}{ }^{6}$ sur chaque mot lexical de la séquence : brousse, pleine, brousse, avec, animaux et s'oppose à la figure 2 où on observe un abaissement tonal caractéristique du bambara (Dumestre, 2003) : [pris la] voi[ ture et] puis [il la] vendue avec une remontée de la $\mathrm{F}_{0}$ sur la dernière syllabe de vendue. Dans les deux cas cependant, l'amplitude des variations mélodiques est tout à fait remarquable avec des pics accentuels qui font intervenir non seulement la $\mathrm{F}_{0}$, mais également l'intensité. La prosodie des locuteurs non reconnus, en revanche, est plus plate avec un écrasement tonal sensible.

Pour conclure, nous pouvons affirmer que les commentaires des auditeurs se sont pour la plupart avérés pertinents et qu'il existe en effet un lien réel entre la phonologie de la L1 du locuteur et sa phonologie en français. Les deux locuteurs les mieux reconnus (7 et 13) le sont sur la base de caractéristiques segmentales et prosodiques, les deux facteurs se renforçant mutuellement. D'autres tests mieux contrôlés s'imposent cependant afin de mettre en place une hiérarchie de degrés d'accent et pour déterminer l'importance des différents facteurs segmentaux par rapport aux facteurs prosodiques.

\section{Identification de la L1 et facteurs sociolinguistiques}

\subsection{Identification de la L1}

Bien que la plupart des auditeurs aient répondu par l'affirmative à la question : «Pensez-vous pouvoir identifier la L1 d'un locuteur à travers son français? », la tâche s'est avérée ardue, comme cela ressort du taux d'identification des L1: 
Tableau 2. Taux d'identification des $\mathrm{L}^{7}$

\begin{tabular}{|l|l|l|l|l|}
\hline L 1 & $\begin{array}{l}\text { Nombre } \\
\text { de } \\
\text { locuteurs }\end{array}$ & $\begin{array}{l}\text { Nombre } \\
\text { d'identifications } \\
\text { possibles }\end{array}$ & $\begin{array}{l}\text { Nombre } \\
\text { d'identifications } \\
\text { réussies }\end{array}$ & $\begin{array}{l}\text { Taux } \\
\text { de } \\
\text { réussite }\end{array}$ \\
\hline Songhay & 3 & 18 & 7 & $39 \%$ \\
\hline Tamasheq & 3 & 18 & 6 & $33 \%$ \\
\hline Bambara & 4 & 24 & 7 & $29 \%$ \\
\hline Sénoufo & 2 & 12 & 0 & $0 \%$ \\
\hline Fulfulde & 2 & 12 & 0 & $0 \%$ \\
\hline Total & 14 & 84 & 20 & $24 \%$ \\
\hline
\end{tabular}

Le nombre d'identifications possibles (nombre de loc. x 6 aud.) est partout largement supérieur au nombre d'identifications correctes (par ex. pour le songhay : 18/7). Le taux de réussite va de 39 à $0 \%$, les deux langues les mieux reconnues étant le songhay et le tamasheq (39 et $33 \%$ ). Cependant, si on met les réponses en rapport avec le profil des locuteurs, on se rend compte que les taux d'identification coïncident avec certains paramètres sociaux. Nous verrons que cela est particulièrement net pour les deux langues non identifiées, le sénoufo et le fulfulde. Le taux moyen de réussite $(24 \%)$ confirme la tendance générale d'un écart entre capacité déclarée et capacité réelle à identifier les accents - qu'il s'agisse d'accents régionaux ou d'accents d'une autre langue (FLE, FLS). L'existence de variétés de français définies en premier lieu selon l'accent du substrat africain peut donc sembler incertaine. Nous avons néanmoins observé en 4 l'existence d'interférences phonologiques réelles ainsi qu'une conscience métalinguistique de la part des auditeurs maliens sur certains points précis, même si les traits identifiés appartiennent à des groupes de langues plutôt qu'à des L1 individuelles.

Les justifications des auditeurs ont en effet introduit un aspect imprévu au départ : les accents régionaux. Raisonnant spontanément en termes de " nordistes » et de "sudistes », ils hésitent le plus souvent entre des langues appartenant effectivement à ces deux zones. Le fulfulde, parlé au centre, est placé au nord par tous (y compris les deux auditeurs de L1 fulfulde), même si certains mentionnent qu'il se situe au centre. Personne ne l'associe au sud. Le sud-est (région du sénoufo) est mentionné dans 4 des 84 réponses ; nous l'avons classé avec le sud. Aucun des locuteurs ne parle une langue de l'ouest, et aucun auditeur n'a non plus évoqué cette région. En conséquence, nous avons décidé d'examiner les réponses selon le paramètre géographique, en adoptant la distinction nord / sud.

Tableau 3. Taux d'identification de la région de la $\mathrm{L}^{8}$

\begin{tabular}{|l|l|l|l|l|l|}
\hline L1 & Nord & Sud & $\begin{array}{l}\text { Nombre } \\
\text { d'identifications } \\
\text { possibles }\end{array}$ & $\begin{array}{l}\text { Nombre } \\
\text { d'identifications } \\
\text { réussies }\end{array}$ & $\begin{array}{l}\text { Taux de } \\
\text { réussite }\end{array}$ \\
\hline Tamasheq & $\mathrm{x}$ & & 18 & 17 & $94 \%$ \\
\hline Sénoufo & & $\mathrm{X}$ & 12 & 9 & $75 \%$ \\
\hline Bambara & & $\mathrm{X}$ & 24 & 15 & $63 \%$ \\
\hline Songhay & $\mathrm{x}$ & & 18 & 9 & $50 \%$ \\
\hline Fulfulde & $\mathrm{x}$ & & 12 & 2 & $17 \%$ \\
\hline
\end{tabular}




\begin{tabular}{|l|l|l|l|l|l|}
\hline Total & & & 84 & 52 & $62 \%$ \\
\hline
\end{tabular}

Dès que l'on prend la zone géographique en considération, les résultats changent: le score moyen s'améliore radicalement, avec $62 \%$ d'identifications réussies. Le tamasheq est reconnu comme étant du nord à $94 \%$, et le sénoufo, qui n'avait été identifié par aucun auditeur, est situé au sud dans $75 \%$ des réponses. Il est évidemment plus facile d'identifier un ensemble géographique qu'un point précis, comme le constatent d'ailleurs Bauvois, Boughton et aussi Woehrling et Boula de Mareüil. . . Cependant, le score au Mali n'est pas fortuit: nous avons vu qu'il repose sur des caractéristiques linguistiques mises en évidence tant par les linguistes que par les réalisations des locuteurs et les commentaires métalinguistiques des auditeurs.

Tous les auditeurs donnent l'accent du nord comme l'accent marqué ${ }^{10}:$ " Parce qu'il y a certaines ethnies où tout de suite on sait, le peul [fulfulde], par exemple, le songhay, le tamasheq. Les ethnies du nord on reconnaît facilement. Cette personne manque les traits du nord» (à propos de la loc. 1, sénoufo). La confusion des 3 langues du nord (plus systématique qu'entre les langues du sud) confirme l'existence, dans l'imaginaire des Maliens, d'une variété propre à cette région. Ils confondent en particulier songhay / tamasheq (8 réponses) et songhay / fulfulde (6), plus rarement tamasheq / fulfulde (1). Le rôle central du songhay (qui était aussi la langue la mieux reconnue, cf. tableau 2) est instructif, car son caractère mixte est souvent mis en avant (par ex. par Nicolaï, 1989). Son rôle de lingua franca au nord peut aussi contribuer à sa dominance dans ce test. Nous verrons cependant en 5.2 que l'accent marqué des deux locuteurs songhay s'explique aussi par des facteurs sociolinguistiques, qui sont également déterminants pour la non reconnaissance du fulfulde (L1 de deux des auditeurs, qui n'ont pas reconnu leur propre L1).

Si la reconnaissance régionale est meilleure que celle de la $\mathrm{L} 1$, nos résultats ne coulent pas tous les locuteurs d'une même région dans un moule fixe, pas plus que les locuteurs d'une même L1. Certains sont beaucoup mieux reconnus que d'autres et on se rend compte que les taux d'identification coïncident avec certains facteurs extralinguistiques propices ou non au nivellement de l'accent. Les trois facteurs qui se sont révélés déterminants sont : le niveau d'études, la durée du séjour à Bamako et l'exposition au français. Ils feront l'objet de la discussion qui suit, avant la présentation de la synthèse.

\subsection{Facteurs sociolinguistiques}

Tableau 4. L'impact du niveau d'études ${ }^{11}$

\begin{tabular}{|l|l|l|l|l|l|}
\hline $\begin{array}{l}\text { Niveau } \\
\text { d'études }\end{array}$ & $\begin{array}{l}\text { No. du } \\
\text { locuteur }\end{array}$ & L1 & $\begin{array}{l}\text { Nombre } \\
\text { d'identifications } \\
\text { de la L1 }\end{array}$ & $\begin{array}{l}\text { Nombre } \\
\text { d'identifications } \\
\text { de la région }\end{array}$ & $\begin{array}{l}\text { Sans } \\
\text { réponse }\end{array}$ \\
\hline CEP & 7 & TA & 4 & 6 & \\
\hline CEP & 13 & BA & 4 & 5 & 3 \\
\hline DEA+2 & 8 & FU & & 1 & 3 \\
\hline Thèse & 14 & FU & & 1 & \\
\hline
\end{tabular}

Le tableau 4 révèle que les deux locuteurs les mieux identifiés (loc. 7 et 13) n'ont que 6 ans d'école (CEP). Leur L1 est identifiée par 4 auditeurs et la région par 6 et 5 auditeurs respectivement. L'un de ces locuteurs est du nord (tamasheq), l'autre du sud (bambara). Ni la L1 ni l'origine géographique n'expliquent donc ces taux élevés de reconnaissance.

Les deux locuteurs les moins bien reconnus ont en revanche le plus haut niveau d'études, DEA +2 et thèse. Il s'agit des deux locuteurs du fulfulde (loc. 8 et 14). Aucun auditeur n'a identifié leur L1 et un seul auditeur a reconnu leur région. Il faut en conclure que c'est le niveau d'études et non la langue fulfulde 
qui explique le problème d'identification, dû au nivellement de l'accent. Comme le dit un auditeur à propos de la loc. 8, fulaphone : «Elle doit être très avancée dans les études. [...] C'est un français assez standard, où il n'y a pas de place pour les particularismes locaux ». Le niveau d'éducation est par ailleurs la caractéristique la plus fréquemment évoquée par les auditeurs dans leurs justifications. L'impact de la scolarisation ressort aussi d'un travail sur l'imaginaire linguistique au Sénégal. Moreau (1998) constate qu'il y a différents imaginaires pour différentes langues : « Pour le français, tous les faits de variation sont ramenés à des différences de scolaristion. [...] Cette association systématique entre qualité de la langue et scolarité donne à penser que la variation n'est jamais pensée autrement qu'en termes d'écart plus ou moins important par rapport à la norme scolaire. » (op. cit. : 116, c'est l'auteur qui souligne).

Entre les deux pôles d'études, thèse et CEP, se trouvent des niveaux oscillant entre DEF et BAC+1,2, sans qu'un lien univoque ne s'établisse entre études et identification de la L1. Parmi d'autres paramètres pouvant influer sur l'accent, nous avons identifié l'itinéraire hors de l'aire d'origine, notamment le séjour à Bamako - l'effet de la ville sur les pratiques langagières étant bien établie (Calvet 1994). Les auditeurs caractérisent en effet souvent l'accent de Bamako comme «neutre».

Tableau 5. L'impact du séjour à Bamako

\begin{tabular}{|l|l|l|l|l|l|}
\hline $\begin{array}{l}\text { Durée du } \\
\text { séjour à } \\
\text { Bamako }\end{array}$ & Itinéraire & $\begin{array}{l}\text { No. du } \\
\text { locuteur }\end{array}$ & L1 & $\begin{array}{l}\text { Nombre } \\
\text { d'identifications } \\
\text { de la L1 }\end{array}$ & $\begin{array}{l}\text { Nombre } \\
\text { d'identifications } \\
\text { de la région }\end{array}$ \\
\hline 6 mois & Né près de Gao en 1982 & 9 & SO & 3 & 3 \\
\hline 1 an & Né près de Gao en 1984 & 5 & SO & 3 & 3 \\
\hline $\begin{array}{l}\text { Env. } 12 \\
\text { ans }\end{array}$ & $\begin{array}{l}\text { Née près de Gao en 1976, } \\
\text { Burkina Faso 3 ans }\end{array}$ & 12 & TA & 1 & 5 \\
\hline 57 ans & $\begin{array}{l}\text { Né à Djenné en 1938, } \\
\text { parents FU, USA 1 an }\end{array}$ & 4 & BA & 1 & 4 \\
\hline Natif & $\begin{array}{l}\text { Né et vit à Bamako depuis } \\
\text { toujours }\end{array}$ & 10 & BA & 0 & 3 \\
\hline Natif & $\begin{array}{l}\text { Né et vit à Bamako depuis } \\
\text { toujours }\end{array}$ & 13 & BA & 4 & 5 \\
\hline
\end{tabular}

D'après le tableau 5 , les locuteurs 5 et 9 , de L1 songhay, se distinguent par le fait d'être récemment arrivés à Bamako (respectivement 6 mois et 1 an). Le nivellement de l'accent normalement observé dans la capitale n'ayant pas eu le temps de se manifester, leur L1 est reconnue par 3 auditeurs, un score qui les place conjointement au $3^{\mathrm{e}}$ rang, juste après les deux locuteurs de niveau CEP. Ce score est obtenu malgré leur niveau d'études élevé : bac +1 ou $2^{12}$. La bonne identification et la saillance de certains traits linguistiques, plus prononcés chez eux que chez les locuteurs de tamasheq, de profils plus diversifiés, doivent donc être interprétées aussi à la lumière des circonstances extralinguistiques.

Pour les autres locuteurs (représentés dans ce tableau par deux d'entre eux, les loc. 12 et 4), le séjour à Bamako varie entre 12 et 62 ans. La durée du séjour paraît sans importance pour le nivellement après quelques années : la loc. 12 (tamasheq) et le loc. 4 (bambara) ont passé respectivement 12 et 57 ans dans la capitale, et dans les deux cas, la L1 n'est reconnue que par un seul auditeur. Comme le constate l'un des auditeurs : «Ceux qui sont venus à Bamako il y a cinq ans, dix ans, il y a une influence, parce que le bambara commence à influencer leur langue. » (à propos du loc. 4 - de parents fulaphones, mais vivant à Bamako depuis 57 ans et citant le bambara comme sa L1).

Qu'en est-il alors des locuteurs natifs ? Leur accent est-il aussi considéré comme neutre ? C'est l'avis d'un auditeur natif de Bamako : «Quand on est né à Bamako, on est né avec cet accent neutre là. ». Or, ce n'est pas nécessairement le cas. Le troisième facteur de nivellement, l'exposition au français, peut 
distinguer des locuteurs de profil semblable. Ainsi, les loc. 10 et 13, natifs de Bamako, ont-ils tous deux le niveau CEP et le bambara pour L1. Alors que le loc. 13 est bien identifié ( 4 auditeurs pour la L1 et 5 pour la région), ce qui le place comme l'un des deux les mieux reconnus, le loc. 10 est l'un des trois les moins bien reconnus (sa L1 n'est reconnue par personne, et la région par 3 auditeurs seulement). Il s'avère que le loc. 10 est planton à l'université et quotidiennement exposé au français, tandis que le loc. 13 , actuellement à la retraite, était chauffeur de duurunin ${ }^{13}$ et donc sans beaucoup d'occasions de pratiquer le français.

La synthèse des 3 facteurs de nivellement montre qu'ils jouent conjointement pour rendre malaisée l'identification des L1. Le tableau 6 fait ressortir (en gras) ces facteurs pour les 4 locuteurs dont la L1 n'a pas été reconnue :

Tableau 6. Synthèse des facteurs de nivellement de l'accent

\begin{tabular}{|l|l|l|l|l|l|}
\hline $\begin{array}{l}\text { No. du } \\
\text { locuteur }\end{array}$ & L1 & $\begin{array}{l}\text { Identifications } \\
\text { de la L1 / la } \\
\text { région }\end{array}$ & $\begin{array}{l}\text { Niveau } \\
\text { d'études }\end{array}$ & $\begin{array}{l}\text { Séjour à Bamako } \\
\text { + voyages }\end{array}$ & $\begin{array}{l}\text { Exposition au } \\
\text { français }\end{array}$ \\
\hline 8 & FU & $0 / 3$ & DEA+2 & 22 ans + 10 ans en France & SOUVENT \\
\hline 14 & FU & $0 / 3$ & Thèse & $\begin{array}{l}\mathbf{3 3} \text { ans }+\mathbf{5} \text { ans en Union } \\
\text { Soviétique }\end{array}$ & SOUVENT \\
\hline 2 & SE & $0 / 4$ & $\begin{array}{l}\text { Bac (DEF+4, } \\
\text { comptabilité) }\end{array}$ & $\begin{array}{l}\text { 28 ans + multiples } \\
\text { mutations au Mali }\end{array}$ & SOUVENT \\
\hline 1 & SE & $0 / 5$ & $\begin{array}{l}\text { DEF (+2, CAP, } \\
\text { jardin } \\
\text { d'enfants) }\end{array}$ & $\begin{array}{l}\text { Env. 30 ans + multiples } \\
\text { mutations au Mali }\end{array}$ & SOUVENT \\
\hline
\end{tabular}

Les 4 locuteurs dont la L1 n'a pas du tout été reconnue parlent 2 langues typologiquement très différentes (avec et sans tons), sénoufo et fulfulde. Mais ils ont en commun de longs séjours en dehors de leur aire d'origine : entre 22 et 33 ans à Bamako, plus 5 ou 10 ans à l'étranger ou bien de multiples mutations à l'intérieur du pays, et tous pratiquent régulièrement le français au travail. Les deux locuteurs du fulfulde ont, de plus, le niveau le plus élevé d'études, thèse et DEA+2. Aussi le score de reconnaissance est-il encore plus faible pour le fulfulde que pour le sénoufo si on prend en compte les identifications de la région : 3 pour chacun des locuteurs de fulfulde, contre 4 et 5 pour les locuteurs du sénoufo - qui sont de niveau d'études moins élevé (Bac et DEF).

Le nivellement de l'accent par les facteurs sociaux est donc considérable. Dans le contexte malien, le plus important est le niveau de scolarisation, dont découlent en partie les autres facteurs. En effet, une scolarisation poussée ouvre la porte aux bourses, à des postes à l'étranger ou dans la fonction publique, situations où on est souvent exposé au français.

\section{Conclusion}

Notre étude sur la perception d'accent porte sur le FLS qui, rappelons-le, se distingue du FL1, du FL2 et du FLE par son mode d'appropriation. A notre connaissance, cette étude est la première sur le FLS qui confronte les représentations des auditeurs au signal acoustique et aux caractéristiques phonologiques des L1 des locuteurs.

Une comparaison des commentaires des auditeurs aux performances des locuteurs a mis en évidence chez les auditeurs une aptitude certaine à définir les traits discriminants des différents accents. Nous avons vu en particulier que si la prononciation des voyelles antérieures arrondies est problématique, elles sont plus ou moins bien maîtrisées selon la L1 du locuteur et du niveau d'études, et que les locuteurs tendent à 
projeter sur le français la prosodie de leur L1. La chute variable du R mérite des études plus approfondies dans d'autres variétés de français africain puisque sur la base de nos résultats, nous formulons l'hypothèse que le degré de chute sera proportionnel au nombre de consonnes autorisées en coda dans la L1. Les facteurs sociolinguistiques viennent cependant nuancer la présence même des traits discriminants: l'influence de la L1 sur le français s'atténue proportionnellement au niveau d'études, à la mobilité géographique et à l'exposition au français. La prépondérance du facteur éducatif, qui s'explique par le mode d'appropriation du français, se traduit tant par les discours épilinguistiques, révélateurs des représentations des auditeurs, que par leur perception des L1 et la mesure acoustique des réalisations.

Ces résultats auront un impact sur la notion même de variétés africaines de français. Les données que nous avons explorées indiquent en effet, au-delà de l'influence certaine de facteurs sociolinguistiques sur les variétés locales de français, que l'étiquette «français du Mali » cache une pluralité d'idiomes dans lesquels les structures linguistiques de la L1 jouent un rôle de premier plan.

\section{Références bibliographiques}

Bauvois, C. (1996). Parle-moi et je te dirai peut-être d'où tu es. Revue de Phonétique Appliquée, 121, 291-309.

Boughton, B. (2006). When Perception isn't Reality : Accent identification and perceptual dialectology in French. Journal of French Language Studies, 16, 277-304.

Bordal, G. (2006). Traces de la créolisation dans un français régional. Le cas du /R/à l'île de la Réunion, mémoire de master, Université d'Oslo. (www.projet-pfc.net).

Boula de Mareüil, Ph. et B. Vieru-Dimulescu (2006). The Contribution of Prosody to the Perception of Foreign Accent. Phonetica, $63: 1,1-21$.

Boutin, B. A., C. Lyche et G. Prignitz (2007). PFC en terre africaine. Bulletin PFC 7, www.projet-pfc.net.

Boutin, B. A. et G. Turcsan (2009). La prononciation du français en Afrique : la Côte d'Ivoire. In J. Durand, B. Laks et C. Lyche (éds.). Phonologie, variation et accents du français. Paris : Hermès, 131-152.

Calvet, L.-J. (1994). Les voix de la ville. Introduction à la sociolinguistique urbaine. Paris, Payot.

Canut, C. et G. Dumestre (1993). Français, bambara et langues nationales au Mali, in D. de Robillard, et M. Beniamino (éds.). Le français dans l'espace francophone. Paris : Champion, tome 1, 219-228.

Carlson, R. (1994). A Grammar of Supyire. Berlin : Mouton de Gruyter.

Cissé, I. (2009). Comparaison de deux langues en contact, le fulfulde et le bambara, dans une perspective typologique: structures phonémiques, syllabiques et lexicales. Mémoire de master II, Université Stendhal Grenoble III.

Cuq, J.-P. (1991). Le français langue seconde: origines d'une notion et implications didactiques. Paris : Hachette.

Dister, A.., F. Gadet, R. Ludwig, C. Lyche, L. Mondada, S. Pfänder, I. Skattum (2008). Deux nouveaux corpus internationaux du français: CIEL-F (Corpus International et Ecologique de la Langue Française) et CFA (Français contemporain en Afrique et dans l'Océan Indien). Revue de linguistique romane, nos. 285-286, janvier-juin, vol. $72,295-314$.

Dumestre, G. (2003). Grammaire fondamentale du bambara. Paris : Karthala.

Durand, J. et C. Lyche. (2003). Le projet 'Phonologie du Français Contemporain' (PFC) et sa méthodologie. E. Delais et J. Durand (éds.). Corpus et variation en phonologie du français : méthodes et analyses. Toulouse : Presses Universitaires du Mirail, 212-276.

L'état de l'Afrique 2009 (2009). Paris Jeune Afrique, hors série no. 21.

Féral, C. de (1997). Formes de français parlé à Yaoundé. LACITO Documents, 10, 37-43.

Gadet, F. (2007). La variation sociale en français. Nouv. éd. revue et augmentée. Paris : Ophrys. 
Guion, S. G., J. E. Flege et J. D. Loftin (2000). The Effect of L1 use on pronunciation in Quichua-Spanish bilinguals. Journal of Phonetics, 28, 27-42.

Heath, J. (2005). A Grammar of Tamashek (Tuareg of Mali). Berlin, New York : Mouton de Gruyter.

Lyche, C. et I. Skattum (2009). Unité et diversité du français contemporain en Afrique et dans l'Océan Indien. Communication au Colloque International La variation du français dans les aires créolophones et francophones, 3-6 juin 2009, Université de la Réunion (à paraître).

Manessy, G. (1994). Le français en Afrique noire. Mythe, stratégies, pratiques. Paris : L'Harmattan.

Moreau, M.-L., (1998). De l'imaginaire linguistique à la politique linguistique. A la recherche d'un standard pour le diola (Casamance, Sénégal), in C. Canut (éd.). Attitudes, représentation et imaginaires linguistiques en Afrique: Quelles notions pour quelles réalités? Paris : INALCO.

Moreau, M.-L., Nd. Thiam et C. Bauvois (1998). Le marquage identitaire dans le français d'Afrique. Etude explorative au Sénégal. In L.-J. Calvet et M.-L. Moreau (éds.). Une ou des normes? Insécurité linguistique et normes endogènes en Afrique francophone. Paris : Didier Erudition, 11-127.

Nicolaï, R. (1981). Les dialectes du songhay. Contribution à l'étude des changements linguistiques. Paris : SELAF.

Nicolaï, R. (1989). Songhay et mandé, Mandenkan, 18, 69-80.

Piske, Thorsten, I. R. A. MacKay et J. E. Flege (2001). Factors Affecting Degree of Foreign Accent in an L2 : a review. Journal of Phonetics, 29, 191-215.

Prignitz, G. (1997). Le normal et le normatif. Bulletin du Centre d'étude des plurilinguismes, Université de Nice, no. spéc. « A propos du français en Afrique, questions de norme », 59-87.

Queffélec, A. (2008). L'évolution du français en Afrique noire, pistes de recherche. In K. Holter et I. Skattum (éds.). La francophonie aujourd'hui. Réflexions critiques. Paris : L'Harmattan.

Rossillon, Ph. (1995). Atlas de la langue française. Paris : Bordas.

Skattum, I. (2008). Mali : In Defence of Cultural and Linguistic Pluralism. In A. Simpson (éd.). Language \& National Identity in Africa. Oxford : Oxford University Press, 98-121.

Vieru-Dimulescu, B. et Ph. Boula de Mareüil (2006). Perceptual Identification and Phonetic Analysis of 6 Foreign Accents in French, Interspeech 2006, Pittsburg, Pennsylvania, Ninth International Conference on Spoken Language Processing, International Speech Communication Association (ISCA).

Woehrling, C. et Ph. Boula de Mareüil (2006). Identification d'accents régionaux en français : perception et analyse. Revue PArole, 37, 25-65.

\footnotetext{
${ }^{1}$ Le classement est toutefois incertain, cf. Nicolaï (1989).

2 www.projet-pfc.net.
}

${ }^{3}$ Si l'âge d'appropriation de la L2 s'impose comme un facteur déterminant pour la notion d'accent dans les études portant sur l'acquisition (naturelle) de la L2 (Piske et al. 2001), ce paramètre ne saurait jouer dans le contexte africain puisque l'appropriation du français se fait principalement - et dans un pays comme le Mali quasi exclusivement - par le biais de l'école, donc à peu près au même âge pour tous.

${ }^{4}$ D'autres niveaux d'instruction (écoles professionnelles, études sans sanction, diplômes anciens, etc.) ont été standardisés et classifiés dans ces trois catégories.

${ }^{5}$ Rappelons que le français est une langue où l'accent possède une fonction purement démarcative, indiquant la frontière droite d'un groupe et non d'un mot. A la différence, en tamasheq, songhay et fulfulde, l'accent est associé à l'unité lexicale.

${ }^{6}$ Les pics de fréquence fondamentale indiquent le plus souvent des pics accentuels. 


\footnotetext{
${ }^{7}$ La L1 est considérée comme identifiée si elle est mentionnée seule, et aussi si l'auditeur propose une alternative, par exemple «tamasheq ou songhay » pour un locuteur de l'une de ces deux langues. Si l'auditeur répond " songhay » pour un locuteur du tamasheq, la réponse est par contre considérée fautive.

${ }^{8}$ La région est considérée comme identifiée si l'auditeur fait explicitement mention de la région, et aussi si la L1 proposée appartient à cette région, même s'il y a confusion des L1 (tamasheq au lieu de songhoy, par ex.).

${ }^{9} \mathrm{Au}$ Sénégal, les auditeurs ont proposé plusieurs régions, ce qui pourrait expliquer le taux plus modeste $(46,7 \%)$ de reconnaissance.

${ }^{10}$ Rappelons qu'aucun des auditeurs ne parle une langue du nord. Cependant, notre analyse montre que les L1 des auditeurs ne semblent avoir aucun impact sur la reconnaissance des L1 des locuteurs.

${ }^{11}$ Le nombre d'identifications de la région comprend celle de la $\mathrm{L} 1$ et de la région. On ne peut donc pas faire l'addition des deux colonnes. "Sans réponse» comprend les réponses du type «J'ai pas pu reconnaître sa langue maternelle». Les réponses fautives ne sont pas comprises dans ce tableau. On peut les déduire en additionnant les deux dernières colonnes. Le loc. 14, par ex., est correctement situé au nord par 1 aud., alors que 3 aud. sont «sans réponse ». Il s'ensuit que 2 aud. l'ont situé au sud, à tort.

${ }^{12}$ Les lycées régionaux sont récents, ce qui explique que ces deux jeunes Songhays ont pu quitter le nord après le $\mathrm{Bac}$, alors que les locuteurs plus âgés sont partis de leur région après le CEP ou le DEF.

${ }^{13}$ Voiture bâchée utilisée comme transport en commun.
} 